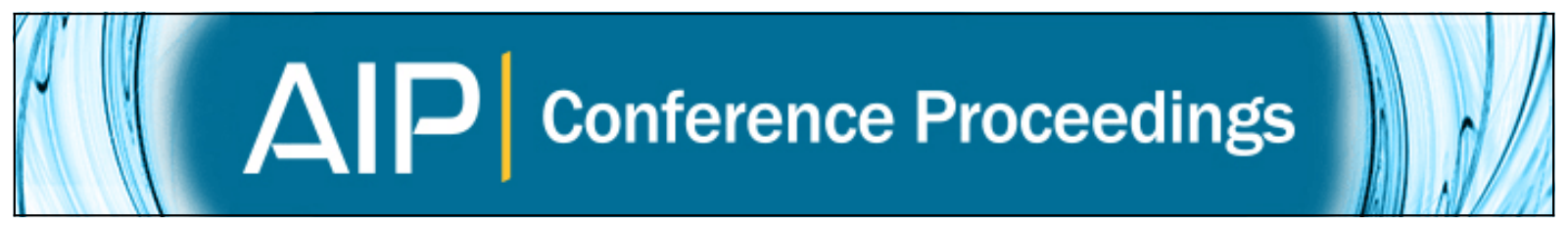

\title{
A mathematical model of phosphorylation AKT in Acute Myeloid Leukemia
}

Y. A. Adi, F. A. Kusumo, L. Aryati, and M. S. Hardianti

Citation: AIP Conference Proceedings 1723, 030001 (2016); doi: 10.1063/1.4945059

View online: http://dx.doi.org/10.1063/1.4945059

View Table of Contents: http://scitation.aip.org/content/aip/proceeding/aipcp/1723?ver=pdfcov

Published by the AIP Publishing

Articles you may be interested in

Colour image segmentation using unsupervised clustering technique for acute leukemia images

AIP Conf. Proc. 1660, 090038 (2015); 10.1063/1.4915882

A model with competition between the cell lines in leukemia under treatment

AIP Conf. Proc. 1637, 1325 (2014); 10.1063/1.4907298

Parameter analysis for a mathematical model of the immune system in leukemia

AIP Conf. Proc. 1637, 1307 (2014); 10.1063/1.4907296

Periodicity in cell dynamics in some mathematical models for the treatment of leukemia

AIP Conf. Proc. 1493, 446 (2012); 10.1063/1.4765527

A delay differential equations mathematical model for the immune response in leukemia AIP Conf. Proc. 1493, 67 (2012); 10.1063/1.4765470 


\title{
A Mathematical Model of Phosphorylation AKT in Acute Myeloid Leukemia
}

\author{
Y.A. Adi ${ }^{1,2, \text { a) }}$, F.A. Kusumo ${ }^{2}$, L. Aryati $^{2}$, M.S. Hardianti ${ }^{3}$ \\ ${ }^{1)}$ Departement of Mathematic Faculty of MIPA Universitas Ahmad Dahlan \\ ${ }^{2)}$ Departement of Mathematic Faculty of MIPA Universitas Gadjah Mada \\ ${ }^{3)}$ Department of Internal Medicine, Faculty of Medicine, Universitas Gadjah Mada \\ ${ }^{\text {a)} C o r r e s p o n d i n g ~ a u t h o r ~: ~ y u d i . a d i @ ~ m a t h . u a d . a c . i d ~}$
}

\begin{abstract}
In this paper we consider a mathematical model of PI3K/AKT signaling pathways in phosphorylation AKT. $\mathrm{PI}$ 3K/AKT pathway is an important mediator of cytokine signaling implicated in regulation of hematopoiesis. Constitutive activation of PI3K/AKT signaling pathway has been observed in Acute Meyloid Leukemia (AML) it caused by the mutation of Fms-like Tyrosine Kinase 3 in internal tandem duplication (FLT3-ITD), the most common molecular abnormality associated with AML. Depending upon its phosphorylation status, protein interaction, substrate availability, and localization, AKT can phosphorylate or inhibite numerous substrates in its downstream pathways that promote protein synthesis, survival, proliferation, and metabolism. Firstly, we present a mass action ordinary differential equation model describing AKT double phosphorylation (AKTpp) in a system with 11 equations. Finally, under the asumtion enzyme catalyst constant and steady state equilibrium, we reduce the system in 4 equation included Michaelis Menten constant. Simulation result suggested that a high concentration of PI3K and/or a low concentration of phospatase increased AKTpp activation. This result also indicates that PI3K is a potential target theraphy in AML.
\end{abstract}

Keywords : Acute Myeloid Leukemia, PI3K, AKT, Mathematical Model

\section{INTRODUCTION}

Acute Myeloid Leukemia (AML) is a hematological disorder characterized by deregulated proliferation of myeloid cells immature. In this disease, hematopoietic stem cell differentiation is blocked which resulted in the accumulation of neoplastic cells in the bone marrow of blasts [1 - 2]. AML patients can be stratified into three risk categories favourable, intermediate, and unfavourable using Cytogenetics and FISH (Fluorescence In Situ Hybridization). Leukemogenesis in AML can be caused by mutations in NPM1, CEBPA, FLT3-ITD. AML with mutations on receptors fms like tyrosine kinase-3 (FLT3) on the domain Juxtamembrane (JM) internal tandem duplication (ITD) (FLT3-ITD) is very aggressive and have a poor prognosis [3 - 4], while mutation in CEBPA and NPM1 have good outcomes. In addition, AML can also be caused by a type of epigenetic mutations in IDH1, IDH2, DNMT3A, and TET2. Several other types of mutations are also common. It is also often found several mutations that occur together. Mutations of DNA methyltransferase 3A (DNMT3A) is the most common somatic mutations occur after FLT3-ITD mutation. Almost all mutations in AML has a poor prognosis, except CEBPA and NPM1 mutations without FLT3-ITD [5].

In the process of hematopoiesis regulation, PI3K/AKT signalling pathways is very important role. Most cases of AML showed deregulated activity of the PI3K/AKT/mTOR signal transduction [6]. In normal conditions, FLT3 induces phosphorylation and activation of molecules in the cytoplasm, which is the PI3K regulator subunit P85 and catalytic subunit P110 from Phospoinositol-3-kinase (PI3K), Ras GTPase, phospoliphase C- $\gamma$, Shc, Grb2, and Src tyrosine kinase which results in phosphorylation of the protein. Activation is followed by the next downstream pathways, the PI3K/Protein Kinase B (PKB) or known as AKT and mitogen-activated protein kinase (MAPK) pathway.

FLT3-ITD mutation results in loss of normal function of FLT3 that do not normally have an impact on the function of PI3K/AKT pathway and RAS/MAPK/ERK pathway. In addition, this mutation also activates STAT5 pathway [4]. On PI3K/AKT pathway, activation or phosphorilation of AKT excess occurred which resulted in the 
regulation of apoptosis and inhibition of the growth of abnormal cells that uncontrollably (AML blast). Constitutive activation of AKT also phosphorylated and inhibit the transcription factor FOXO3a [7] that caused its inactive [8]. FOXO3a is a transcription factor that plays an important role in the process of apoptosis and cell cycle regulation.

There are many mathematical and computational model that gives an overview activity of signal transduction pathways. Hatakeyama [9], Tan et al [10], has developed a computational model signal transduction pathways in Mitogen Activates Protein Kinase (MAPK ). These models studied signal transduction pathways and its relation to the investigation of therapy in the event of deviation due to certain diseases. Hatakeyama et al developed a computational model of the MAPK and Akt signaling pathways in HRG-induced ErbB signal. The models in the form of system differential equations consist 33 equations that describe the crosstalk between MAPK and PI3K/AKT signalling pathways in the regulation of signal transduction, which showed that the HRG signaling proteins regulated by PP2A posphatase which also modulate protein kinase. Tan et al developed a computational model of Gab1/2 on the activation of AKT in the form of ODE system that consist 71 reaction with 14 different protein and 43 kinetic parameters. Associated with PI3K signaling pathways, Lee et al [11] developing the model proto-oncogene Myc regulation by Erk and PI3K in the model with ERK and PI3K as an input signal and Myc as an output signal.

Referring to the models of signal transduction pathway as mentioned above, in this paper we modeled and simulated the AKT phosphorylation as a result of FLT3-ITD mutations that activate PI3K/AKT signaling pathways in cases of AML. Furthermore, from this model we expected to obtain a description of how to use inhibitors in the right signalling pathways to threat AML.

\section{PI3K/AKT SIGNALING PATHWAY IN AML}

Phospoinositol-3 kinase (PI3K) is a family of enzym that have crucial rule in machanism of cell regulation. The family of PI3K has been categorized into three classes, which class I PI3K is the best understood and are key players of multiple intracellular signaling networks that integrate a wide variety of signals and corelated with hematolgy malignancy. The most important PI3K substrate is phosphatidynositol 4,5 biphosphate (PtdIns $(4,5) \mathrm{P} 2)$ writen as PIP2, which is phophorylated to yield phosphatidylinositol 3,4,5 triphosphate (PtdIns3,4,5P3) written as PIP3. Although there are more than 50 downstream effectors of PI3K, but the most dominant effectors are AKT. AKT is a sherine/threonine protein kinase also known as protein kinase $\mathrm{B}(\mathrm{PKB})$ contains an $\mathrm{NH} 2$ terminal pleckstrin homology (PH) domain, which interacts with PIP3 and phospoinositide dependent kinase-1 (PDK1) at the plasma membrane resulting in AKT phosphorylation at threonine 308. For full activation, AKT also phosphorylation by mechanistic target of rapamycin complex 2 (mTORC2) at serine 473. In AML constitutive activation PI3K/AKT is a common feature, that is about 50 to $70 \%$ of patien with AML display phosphorilation of both Thr 308 and Ser 473 AKT [8]. Constitutive activation or overactivation of PI3K/AKT signaling pathway has been observed in Acute Meyloid Leukemia (AML) it caused by the mutation of fms-like tyrosine kinase 3 in internal tandem duplication (FLT3-ITD), the most common molecular abnormality associated with AML. Downstream effects of this signal included inactivation of transcription factor GSK3 and FOXO3a through phosporylation that caused its retained in the cytoplasma and its transcriptional activity is prevented.

Negative regulator of PI3K/AKT signal is a phosphatase and tensin homologue deleted on chromosome ten (PTEN). PTEN is a protein phosphatase that dephosphorylation the PIP3 back into PIP2. Dephosphorylation is essential to inhibit the signaling pathways of AKT. Loss of PTEN function results in the accumulation of PIP3 and its downstream pathways. Another negative regulator is protein phosphatase 2A (PP2A) that can directly cause dephosphorylation at AKT. Dephosphorylation of AKT can also caused by PH domain leucine-rich repeat protein phosphatase (PHLPP) [6],[12 - 13]. PHLPP can cause dephosphorylation of AKT phosphorylation on one site, which is Ser 473 or in both site site Thr 308 and Ser 473. In AML, the deregulation of the PI3K/AKT pathway is caused due to excessive activation of PI3K, PTEN deletion/ inactive, PP2A deletion/loss. These deregulation caused overactivation of AKT phophorylation that necessary for increased cell survival, proliferation, and leukemic transformation [6]. 


\section{MATHEMATICAL MODEL}

In this model, it is assumed that it had occurred the activation of PI3K caused by mutations of FLT3-ITD . PI3K is further activate PIP2 to form PIP3 . PIP3 activate AKT become AKT phosphorylated on two site, we note with AKTpp mediated by PDK1 and mTORC2 in the process. PIP3 dephosphorylated by PTEN generates PIP2, whereas dephosphorylation AKTpp done by PP2A .The representation of the biochemical reaction shown in Figure 1 below:

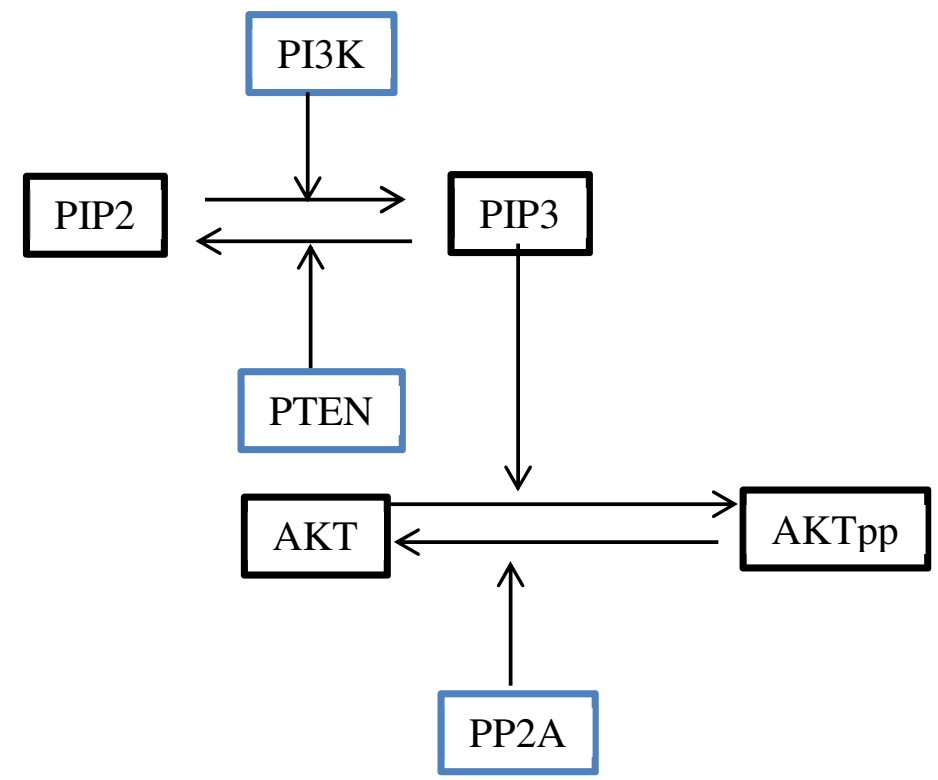

FIGURE 1. Schematic of the biochemical reaction in PI3K/AKT signaling Pathway

From the diagram in Figure 1, the chemical reaction can be divided in two levels of phosphorylation dephosphorylation. The first level biochemical reactions is phosphorylation of PIP2 by PI3K as an enzyme to form PIP3 and dephosphorylation PIP3 by phospatase PTEN back to PIP2, as follows:

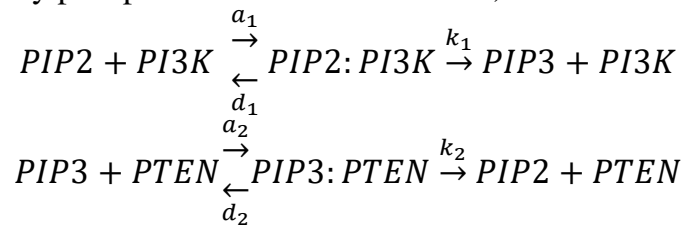

The set of biochemical reactions second level is phosphorylation AKT by PIP3 as enzyme to form AKTpp and dephosphorylation of AKTpp by phospatase PP2A back to AKT, as follows:

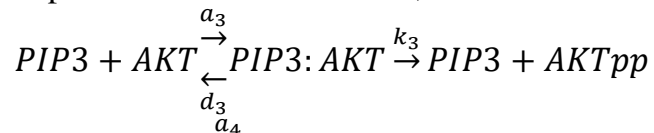

$$
\begin{aligned}
& A K T p p+P P 2 \underset{d_{4}}{\stackrel{a_{4}}{\rightarrow}} A K T p p: P P 2 A \stackrel{k_{4}}{\rightarrow} P P 2 A+A K T
\end{aligned}
$$

where $a_{i}, d_{i}$, and $k_{i}$ is rate of association, dissociation, and activation of protein asumed to be constant.

In order to describe the mathmatical model of biochemical reaction above, we use the law of mass action in an ordinary differential equations (ODE) system. The law of mass action says that the rate of a reaction is proportional to the product of the concentrations of the reactants. Let us define states of the system; : $x_{1}=[\mathrm{PIP} 2]=$ Concentration of PIP2, $x_{2}=$ Concentration of PI3K, $x_{3}=$ Concentration of PIP3, $x_{4}=$ Concentration of PTEN, $x_{5}=$ Concentration of AKT, $x_{6}=$ Concentration of AKTpp, $x_{7}=$ Concentration of PP2A, $y_{1}=$ Concentration of complex PIP2:PI3K, 
$y_{2}=$ Concentration of complex PIP3:PTEN, $y_{3}=$ Concentration of complex PIP3:AKT, $y_{4}=$ Concentration of complex AKTpp:PP2A.

Mathematical model of PI3K/AKT signaling pathway is given by nonlinear ODE system:

$$
\begin{aligned}
\frac{d x_{1}}{d t} & =-a_{1} x_{1} x_{2}+d_{1} y_{1}+k_{2} y_{2} \\
\frac{d x_{2}}{d t} & =-a_{1} x_{1} x_{2}+\left(d_{1}+k_{1}\right) y_{1} \\
\frac{d y_{1}}{d t} & =a_{1} x_{1} x_{2}-\left(d_{1}+k_{1}\right) y_{1} \\
\frac{d x_{3}}{d t}=-a_{2} x_{3} x_{4}+d_{2} y_{2}+k_{1} y_{1}-a_{3} x_{3} x_{5}+\left(d_{3}+k_{3}\right) y_{3} & \frac{d x_{4}}{d t} \\
= & -a_{2} x_{3} x_{4}+\left(d_{2}+k_{2}\right) y_{2} \\
\frac{d y_{2}}{d t} & =a_{2} x_{3} x_{4}-\left(d_{2}+k_{2}\right) y_{2} \\
\frac{d x_{5}}{d t} & =-a_{3} x_{3} x_{5}+d_{3} y_{3}+k_{4} y_{4} \\
\frac{d y_{3}}{d t} & =a_{3} x_{3} x_{5}-\left(d_{3}+k_{3}\right) y_{3} \\
\frac{d x_{6}}{d t} & =-a_{4} x_{6} x_{7}+d_{4} y_{4}+k_{3} y_{3} \\
\frac{d x_{7}}{d t} & =-a_{4} x_{6} x_{7}+\left(k_{4}+d_{4}\right) y_{4} \\
\frac{d y_{4}}{d t} & =a_{4} x_{6} x_{7}-\left(d_{4}+k_{4}\right) y_{4} .
\end{aligned}
$$

In the mechanism of enzyme substract reaction, the enzyme is a catalyst, which only facilitates the reaction, so its total concentration, free enzym plus complex is a constant.This conservation law for the enzyme can be applied for the system (1) - (11), so we can reduce the system in 8 equations, as follow.

On adding the equation (1) and (3) equation, we get

$$
\frac{d x_{2}}{d t}+\frac{d y_{1}}{d t}=0
$$

Integrated with using initial condition $y_{1}(0)=0$, gives

$$
x_{2}=\left[x_{2}\right]_{T}-y_{1} .
$$

Similary, on adding the equation (5) and (6), then (10) and (11), we gets

and

$$
\begin{aligned}
\frac{d x_{4}}{d t}+\frac{d y_{2}}{d t} & =0, \\
x_{4} & =\left[x_{4}\right]_{T}-y_{2} .
\end{aligned}
$$

$$
\begin{aligned}
& \frac{d x_{7}}{d t}+\frac{d y_{4}}{d t}=0, \\
& x_{7}=\left[x_{7}\right]_{T}-y_{4} .
\end{aligned}
$$

Using equation (12) to (14), we can eliminates $x_{2}, x_{4}$, and $x_{7}$ from the system, and we write the system as

$$
\begin{gathered}
\frac{d x_{1}}{d t}=-a_{1} x_{1}\left(x_{2_{T}}-y_{1}\right)+d_{1} y_{1}+k_{2} y_{2} \\
\frac{d y_{1}}{d t}=a_{1} x_{1}\left(x_{2}-y_{1}\right)-\left(d_{1}+k_{1}\right) y_{1} \\
\frac{d x_{3}}{d t}=-a_{2} x_{3}\left(x_{4}-y_{2}\right)+d_{2} y_{2}+k_{1} y_{1}-a_{3} x_{3} x_{5}+\left(d_{3}+k_{3}\right) y_{3}
\end{gathered}
$$




$$
\begin{aligned}
\frac{d y_{2}}{d t} & =a_{2} x_{3}\left(x_{4_{T}}-y_{2}\right)-\left(d_{2}+k_{2}\right) y_{2} \\
\frac{d x_{5}}{d t} & =-a_{3} x_{3} x_{5}+d_{3} y_{3}+k_{4} y_{4} \\
\frac{d y_{3}}{d t} & =a_{3} x_{3} x_{5}-\left(d_{3}+k_{3}\right) y_{3} \\
\frac{d x_{6}}{d t} & =-a_{4} x_{6}\left(x_{7}-y_{4}\right)+d_{4} y_{4}+k_{3} y_{3} \\
\frac{d y_{4}}{d t} & =a_{4} x_{6}\left(x_{7}-y_{4}\right)-\left(k_{4}+d_{4}\right) y_{4}
\end{aligned}
$$

Now, according to the steady state asssumption, the rate of production of enzyme-substract complex must equal the rate of its breakdown. With this assumption we have $\frac{d y_{1}}{d t}=0, \frac{d y_{2}}{d t}=0, \frac{d y_{3}}{d t}=0$, and $\frac{d y_{4}}{d t}=0$, then we eliminate equation (16), (18), (20), and (22) from the system to gets ODE system with 4 equations,

$$
\begin{aligned}
\frac{d x_{1}}{d t} & =-\frac{k_{1} x_{2 T} x_{1}}{K_{1}+x_{1}}+\frac{k_{2} x_{4 T} x_{3}}{K_{2}+x_{3}} \\
\frac{d x_{3}}{d t} & =-\frac{k_{2} x_{4 T} x_{3}}{K_{2}+x_{3}}+\frac{k_{1} x_{2 T} x_{1}}{K_{1}+x_{1}} \\
\frac{d x_{5}}{d t} & =-\frac{k_{3}}{K_{3}} x_{3} x_{5}+\frac{k_{4} x_{7 T} x_{6}}{K_{4}+x_{6}} \\
\frac{d x_{6}}{d t} & =\frac{k_{3}}{K_{3}} x_{3} x_{5}-\frac{k_{4} x_{7 T} x_{6}}{K_{4}+x_{6}}
\end{aligned}
$$

where $K_{1}=\frac{d_{1}+k_{1}}{a_{1}}, K_{2}=\frac{d_{2}+k_{2}}{a_{2}}, K_{3}=\frac{d_{3}+k_{3}}{a_{3}}, K_{4}=\frac{d_{4}+k_{4}}{a_{4}}$ are known as Michaelis-Menten constant.

\section{NUMERICAL SIMULATION}

We now simulate our mathematical model using the ode45 routine generating using Matlab to show the dynamics of PI3K/AKT signaling pathways. Parameters used on this simulation are depicted in table 1 and table 2:

TABLE 1. Parameter value.

\begin{tabular}{cccc}
\hline Parameter & Value & Unit & References \\
\hline$a_{1}$ & $5 \times 10^{-6}$ & Cell mol$^{-1} \mathrm{~s}^{-1}$ & {$[10]$} \\
$a_{2}$ & $5 \times 10^{-6}$ & Cell mol$^{-1} \mathrm{~s}^{-1}$ & {$[10]$} \\
$a_{3}$ & $2.6 \times 10^{-4}$ & Cell mol $^{-1} \mathrm{~s}^{-1}$ & {$[10]$} \\
$a_{4}$ & $1.7 \times 10^{-6}$ & Cell mol$^{-1} \mathrm{~s}^{-1}$ & {$[10]$} \\
$d_{1}$ & 0.1 & $s^{-1}$ & {$[10]$} \\
$d_{2}$ & 0.1 & $s^{-1}$ & {$[10]$} \\
$d_{3}$ & 0.1 & $s^{-1}$ & {$[10]$} \\
$d_{4}$ & 0.1 & $s^{-1}$ & {$[10]$} \\
$k_{1}$ & 0.2 & $s^{-1}$ & {$[10]$} \\
$k_{2}$ & 0.1 & $s^{-1}$ & {$[10]$} \\
$k_{3}$ & 1 & $s^{-1}$ & {$[10]$} \\
$k_{4}$ & 1.5 & $s^{-1}$ & {$[10]$} \\
\hline
\end{tabular}


TABLE 2. Initial Concentration of the protein

\begin{tabular}{llll}
\hline Protein & $\begin{array}{l}\text { Concentration } \\
(\mathbf{n M})\end{array}$ & \multicolumn{1}{c}{ Range $(\mathbf{n M})$} & References \\
\hline PI3K & 10 & $10-100$ & {$[9],[22]$} \\
PTEN & 270 & $0.1-350$ & {$[9],[10]$} \\
PP2A & 150 & $4-150$ & {$[14]$} \\
PIP2 & 700 & $700-800$ & {$[9],[10]$} \\
AKT & 700 & $10-900$ & {$[9],[10],[22]$} \\
\hline
\end{tabular}

Figure 2 shows the simulated protein activities used parameters on table 1 and table 2.

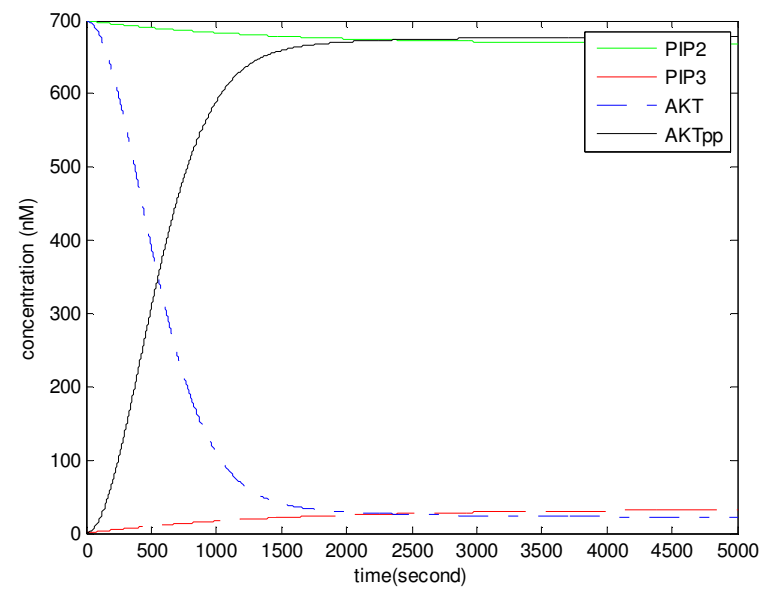

FIGURE 2. Simulated system dynamics of the PI3K/AKT activity.

The initial condition is $[\mathrm{PIP} 2]=700 \mathrm{nM},[\mathrm{PI} 3 \mathrm{~K}]=10 \mathrm{nMl},[\mathrm{PTEN}]=270 \mathrm{nM},[\mathrm{AKT}]=700 \mathrm{nM},[\mathrm{PP} 2 \mathrm{~A}]=150$ $\mathrm{nM},[\mathrm{PIP} 3]=0,[\mathrm{AKTpp}]=0$. With this parameters and initial value, AKTpp reach its maximal level in about 2500 second.

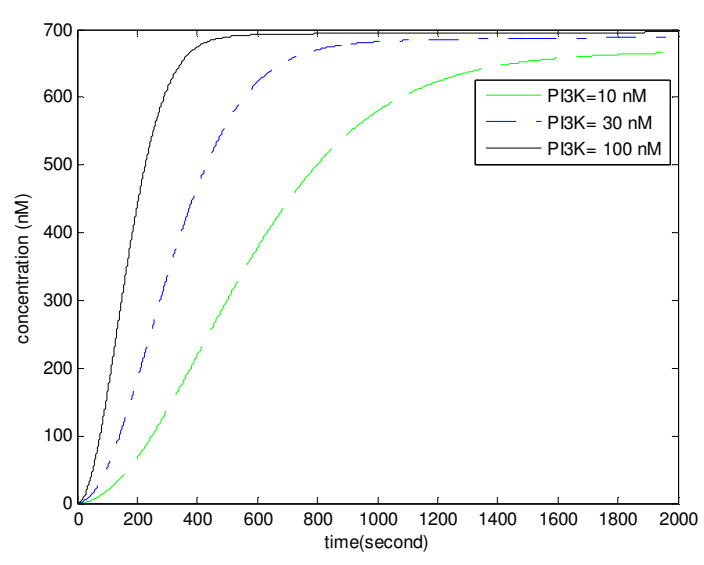

(a)

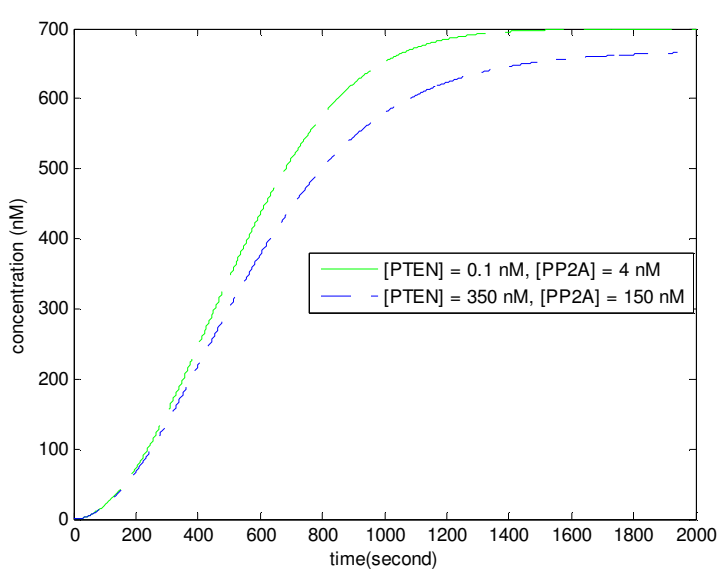

(b)

FIGURE 3. Concentration of AKTpp with different concentrations of PI3K (a) and different concentration of phosphatase (b). 


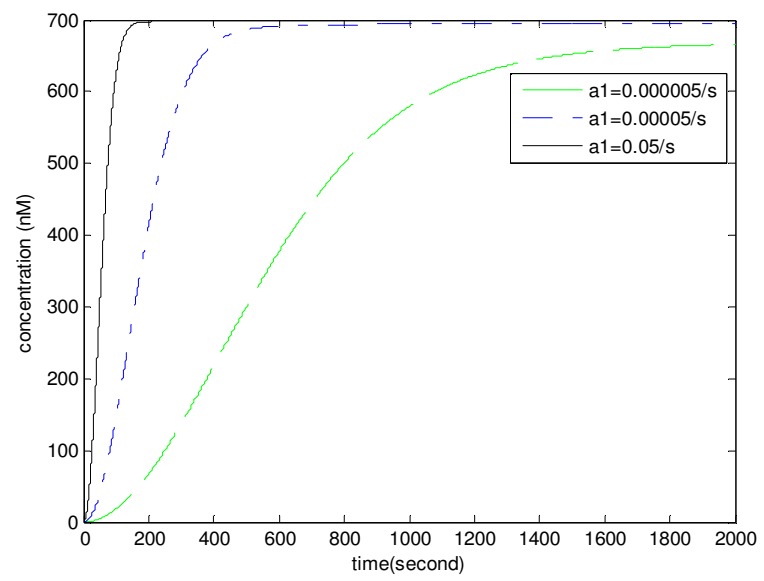

FIGURE 4. Concentration of AKTpp with different reaction rate of PI3K.

It is not known exactly the concentration of AKTpp in normal hematopoiesis regulation. However, it is known that the high concentration of AKTpp, which shows high AKTpp activities, will enhance the development of cell cycle progression. On the other hand overexpression of AKTpp will result in abnormalities of the next signaling pathways that lead to increased antiapoptosis. In the case of AML, overactivity of AKTpp caused due to overactivation of PI3K. Overactivation of PI3K expressed by a high concentration of PI3K or large parameters $a_{1}$. Figure 3a shows the simulated AKTpp when using different concentration of PI3K. We see that when the amount of PI3K is larger, it is corresponding with the larger signal output of AKTpp. Figure 3b shows the simulation AKTpp with different level concentration of phosphatase, PTEN and PP2A. We see that the low concentration of phosphatase caused high lvel of concentration of AKTpp. Figure 4 shows the concentration AKTpp using different value of $a_{1}$, where the greater value of the parameters $a_{1}$ then AKTpp will be even greater. From these figure we see that in a large amount of concentration PI3K or larger parameter $a_{1}$, AKTpp quickly reach its maximum concentration. From the medical view, to reduce the value $a_{1}$ can be done with apply an inhibitor which inhibits PI3K activity.

\section{CONCLUSIONS AND FUTURE WORK}

In this paper we consider a mathematical model of PI3K/AKT signaling pathways in phosphorylation AKT. This model not included protein substract in the downstream pathways of AKTpp. This model also not observe crosstalk with another signal, protein syntesis or degradation. Here, we only provide evidence that overactivation of PI3K caused by FLT3-ITD mutation induced overactivation of AKTpp based on simulation. It is known that conditionally activate AKTpp mediated survival, proliferation, and leukemic transformation of myeloid cells, likely through inactivation of FOXO3a transcriptional activity. In the future work we will observe the downstream pathways of AKTpp, especially the FOXO3a trascription factor that play a critical rule in cellular apoptosis.

\section{REFERENCES}

1. M. P. Martelli,P. Sportoletti, E.Tiacci,M.F. Martelli,B. Falini B ( 2013), Mutation Lanscape of AML with normal Cytogenetics: Biolgical and Clinical Implications, Blood Review 27 (2013) : $13-22$

2. D. B.Rosen, M.D Minden ,S.M. Kornblau,A. Cohen, U. Gayko, et al. (2010) Functional Characterization of FLT3 Receptor Signaling Deregulation in Acute Myeloid Leukemia by Single Cell Network Profiling (SCNP). PLoS ONE 5(10): e13543. doi:10.1371/journal.pone.0013543

3. M. Sharma,S. Mohanty (2013), Molecular Biomarkers in Cytogenetically Normal Acute Myeloid Leukemia: Hanessing the Targets, J. Mol Biomarkers Diagn 2013 S8:009.Doi:10.4172/2155-9929.S8-009

4. S. Takahashi (2011), "Downstream Molecular Pathways of FLT3 in the pathogenesis of Acute Meyloid Leukemia: Biology and Therapeutic Implications", Journal of Hematology and Oncology 2011, 4:13, http://www.jhoonline.org/content/4/1/13 
5. E. Hatzimichael,G.Georgiou,L. Benetatos,E. Briasoulis (2013). Gene Mutations and Molecularly Targeted Therapies in Acute Myloid Leukemia. Am J Blood Res 2013; 3: 29 - 51

6. R. Polak and Buitenhuis (2012). The PI3K/PKB signaling Module as key Regulator of Hematopoiesis : Implications for Therapeutic Strategies in Leukemia. Blood, 26 January 2012, Volume 119, Number 4.

7. C. H. Brandts,B. Sargin,M. Rode, et al (2005) Constitutive Activation of Akt by Flt3 Internal Tandem Duplications is Necessary for Increased Survival, Prpliferation, and Myeloid Tansformation. Cancer Res 2005; 65 (21); $9643-9650$

8. L. Fransecky,L.H. Mochmann,C.D. Baldus (2015). Outlook on PI3K/AKT/mTOR inhibition in Acute Leukemia. Molecular and Cellular Therapies 2015;3:2

9. M. Hatakeyama,S. Kimura,T. Naka,T. Kawasaki,N.Yumoto,M. Ichikawa,J.H. Kim,K. Saito,M. Saeki,M. Shirouzu,S. Yokoyama, \&A. Konagaya ( 2003), “ A Computational Model on The Modulation of Mitogen Activated Protein Kinase (MAPK) and AKT Pathways in Hergulin Induced ErbB Signalling", Biochem J. (2003), 373, $451-463$.

10. W. H.Tan,A.S. Popel,F. MacGabhann (2013)," Computational Model of Gab 1/2 Dependent VEGFR2 Pathway to AKt Activation", PLoS ONE 8 (6):e67438. DOI"10.1371/journal.pone.0067438

11. T. Lee,G. Yao,J. Nevins,L. You (2008)," Sensing and Integrating of ERK and PI3K Signal by Myc”, PLoS Comput Biol. 4 (2):e1000013,DOI:101371/journal.pcbi.1000013

12. A. M. Martelli,M. Nyakern,G. Tabellini,R. Bortul,P.L. Tazzari,C. Evangelisti, and L. Cocco ( 2006). Phospoinositide 3-kinase/Akt Signaling Pathway and Its Therapeutical Implications for Human Acute Myeloid Leukemia. Leukemia (2006), 20 , pp: 911 - 928 DOI:10.1038/sj.leu.2404245

13. G. Wang and T. Li (2014). Computer Aided Targeting of The PI3K/AKT/mTOR Pathway: Toxicity Reduction and Therapeutic Opportunities. Int. J. Molecular.Sciences 2014, 15, 18856 18891;DOI:10.3390/ijms151018856

14. G. Koh,H.F.C Teong ,M.V. Clement,D. Hsu,P.S. Thiagarajan (2006). A decomposition approach to parameter estimation in pathway modeling : a case study of the Akt and MAPK pathways and their crosstalk. Bioinformatic Vol 22 no. 14 : e271 - e280 